1 Universidade Estadual de Montes Claros (Unimontes) - Montes Claros (MG), Brasil.

keniasoutomoreira@hotmail. com

2 Universidade Estadual de Montes Claros (Unimontes). Departamento de Enfermagem e Programa de Mestrado Profissional em Cuidado Primário em Saúde - Montes Claros (MG), Brasil.

di.vieira49@gmail.com

3 Universidade Estadual de Montes Claros (Unimontes). Departamento de Odontologia e Programa de Mestrado Profissional em Cuidado Primário em Saúde - Montes Claros (MG), Brasil.

smelocosta@gmail.com

\section{Qualidade da Atenção Básica: avaliação das Equipes de Saúde da Família}

\author{
Quality of Primary Care: assessment of the Family Health Teams
}

Kênia Souto Moreira', Maria Aparecida Vieira ${ }^{\mathbf{2}}$, Simone de Melo Costa ${ }^{\mathbf{3}}$

RESUMO Objetivou-se analisar a qualidade da Atenção Básica pelas Equipes de Saúde da Família. Utilizaram-se dados da Autoavaliação para Melhoria do Acesso e da Qualidade da Atenção Básica e do Sistema de Informação da Atenção Básica. Foi analisado o padrão de qualidade conforme equipe urbana/rural, composição profissional e cadastro de agravos/doenças por equipe. Ao todo, 75 equipes participaram deste estudo, das quais, 44\% alcançaram padrão satisfatório. Observou-se que equipes mais bem classificadas têm maiores médias de médicos e dentistas $(\mathrm{p}<0,05)$ e que o menor cadastro de pessoas com agravos/doenças não representou melhor qualidade da Atenção Básica. Nota-se, também, que a avaliação de serviços a partir da equipe é importante para a gestão em saúde.

PALAVRAS-CHAVE Atenção Primária à Saúde. Qualidade da assistência à saúde. Saúde pública.

ABSTRACT This study aimed to analyze the quality of Primary Care by Family Health Teams. Data from the Self-Assessment for Improving Access and Quality of Primary Care and Basic Care Information System were used. It was analyzed the quality standard per urban/rural staff, professional composition and records of injuries/illnesses per team. Overall, 75 teams participated in this study, of which, $44 \%$ achieved satisfactory standard. It was observed that best classified teams have higher averages of doctors and dentists $(p<0.05)$ and that the lower register of people with injuries/illnesses did not represent a better quality of Primary Care. It was also noted that service evaluation from the team is important for health management.

KEYWORDS Primary Health Care. Quality of health care. Public health. 


\section{Introdução}

A organização do trabalho na Atenção Básica (AB) é fundamental para que a equipe de Estratégia Saúde da Família (ESF) possa avançar tanto na integralidade da atenção quanto na melhoria do próprio trabalho (BRASIL, 2011). Contudo, as ações das equipes de saúde geralmente são planejadas de forma centralizada e pouco participativa (KAWATA ET AL., 2009). É importante que o planejamento seja enriquecido com o olhar crítico dos atores sociais partícipes da saúde coletiva, gestores, comunidade e profissionais da área. Isso garante a interlocução científica e profissional (SCHRAIBER ET AL., 1999).

Nesse contexto, os processos autoavaliativos na saúde são utilizados como dispositivo de reorganização do trabalho da equipe e da gestão, contribuindo para a melhoria do acesso e da qualidade da $\mathrm{AB}$ em todo o território nacional. Para isso o Ministério da Saúde desenvolve ações de monitoramento e avaliação de processos e resultados. No âmbito do Programa Nacional de Melhoria do Acesso e da Qualidade da Atenção Básica (PMAQ$A B)$, criado pela Portaria $n^{0} 1.654 / 2011$, a autoavaliação da Equipe de Saúde da Família (EqSF) é realizada por instrumento nomeado Autoavaliação para Melhoria do Acesso e da Qualidade da Atenção Básica (AMAQ). A AMAQ avalia padrões de qualidade da $A B$, considerando o grau de atendimento aos padrões estabelecidos no instrumento. Desse modo, por meio da autoanálise, espera-se que os sujeitos avancem na autogestão, na identificação dos problemas e na formulação das estratégias de intervenção para a melhoria dos serviços, das relações e do processo de trabalho na AB (BRASIL, 2011).

No âmbito dos serviços públicos, a gestão em saúde, quando fundamentada na qualidade, implica mudar o foco de atuação e as tecnologias utilizadas. A gestão deve respaldar-se em: resgate da relação interpessoal entre profissional e usuários dos serviços; remuneração, qualificação e capacitação dos profissionais das Equipes de Saúde da Família (EqSFs); qualificação dos gestores e participação ativa de profissionais, no que se refere à pactuação das metas e dos parâmetros de qualidade (SAVASSI, 2012). Este estudo teve como objetivo analisar a qualidade da $\mathrm{AB}$ na visão das EqSFs.

\section{Metodologia}

Trata-se de pesquisa com abordagem quantitativa, desenho transversal e analítico, realizada em cidade polo do norte de Minas Gerais, sendo a quinta maior cidade do estado. Conforme censo demográfico do Instituto Brasileiro de Geografia e Estatística, o contingente populacional do município foi estimado em 394.350 mil habitantes (IBGE, 2015).

A pesquisa foi aprovada pelo Comitê de Ética em Pesquisa envolvendo Seres Humanos, parecer $\mathrm{n}^{\circ} 704.718 / 2014$, e conduzida em conformidade com os princípios éticos contidos na Resolução no 466/2012, da Comissão Nacional de Ética em Pesquisa do Ministério da Saúde. $\mathrm{O}$ projeto de pesquisa contou com a concordância institucional da Secretaria Municipal de Saúde (SMS) do município estudado quanto ao uso de dados das EqSFs.

Os dados de todas as equipes cadastradas no município foram coletados a partir da AMAQ, em site do Ministério da Saúde, impressos e disponibilizados por técnicos da SMS para fins do estudo, em 2014.

A AMAQ, instrumento referente à segunda fase de desenvolvimento do programa de monitoramento e avaliação de qualidade, é uma ferramenta de avaliação de serviços de saúde gerada a partir de instrumentos validados, nacional e internacionalmente, utilizada pelo Ministério da Saúde. $\mathrm{O}$ instrumento apresenta duas dimensões relacionadas à equipe de $\mathrm{AB}$, com análise independente. A primeira trata-se da Unidade Básica de Saúde (UBS), e a segunda, da Educação Permanente, Processo de Trabalho 
e Atenção Integral à Saúde. A segunda dimensão é que foi considerada neste estudo. Cada questão, que compõe a AMAQ, permite avaliar o grau de adequação das práticas da EqSF ao padrão de qualidade apresentado. As questões apresentam uma escala de zero a dez pontos, sendo zero o não cumprimento ao padrão, e 10 a total adequação. Os pontos são somados para classificar a equipe nos seguintes resultados: muito insatisfatório, insatisfatório, regular, satisfatório e muito satisfatório. Quanto maior a pontuação, melhor a classificação da equipe. $\mathrm{O}$ instrumento possibilita identificar os desafios mais críticos, para definir as ações de enfrentamento (BRASIL, 2011).

No Sistema de Informação da Atenção Básica (Siab), setor gestão de dados da SMS do município avaliado, também foram coletadas as seguintes informações pertinentes às EqSFs: localização (urbana, rural); composição e número de profissionais na equipe (enfermagem, medicina, odontologia e agente comunitário de saúde); e número de cadastros de pessoas com agravos/doenças por EqSF (doença de chagas, pacientes com deficiência, hipertensão arterial sistêmica, hanseníase, tuberculose, diabete).

A análise estatística foi realizada por meio do software IBM SPSS 22.0. Realizaramse análises bivariadas para comparar as proporções de equipes em cada padrão de qualidade (muito insatisfatório a muito satisfatório), conforme localização (urbana, rural), pelo teste Likelihood ratio, alternativo do qui-quadrado, de Pearson. Para comparar as médias de profissionais nas equipes entre os padrões de qualidade das equipes, utilizaram-se os testes Anova, seguidos do Bonferroni, para as múltiplas comparações. A correlação entre o total de pontos obtidos pela equipe na dimensão avaliada e o número de profissionais nas EqSFs foi executada por meio da correlação de Pearson. Os testes consideraram o nível de significância p<0,05.

\section{Resultados}

As 75 EqSFs se referem ao total das equipes cadastradas no ano de avaliação do PMAQ$\mathrm{AB}$, correspondendo a $85,3 \%$ na zona urbana. Na dimensão Educação Permanente, Processo de Trabalho e Atenção Integral à Saúde, constatou-se que $44,0 \%$ das equipes alcançaram o nível satisfatório e que nenhuma equipe foi classificada no padrão muito insatisfatório.

Tabela 1. Padrão de qualidade da Atenção Básica na dimensão Educação Permanente, Processo de trabalho e Atenção Integral à Saúde. Equipes de Saúde da Família. Montes Claros, 2014

\begin{tabular}{lrr}
\hline \multicolumn{1}{c}{ Padrão de qualidade da Atenção Básica } & $\mathbf{n}$ & $\%$ \\
\hline Muito insatisfatório & 0 & 0,0 \\
Insatisfatório & 8 & 10,7 \\
Regular & 30 & 40,0 \\
Satisfatório & 33 & 44,0 \\
Muito satisfatório & 4 & 5,3 \\
\hline Total de Equipes de Saúde da Família & 75 & 100,0 \\
\hline
\end{tabular}

Ao associar padrões de qualidade da $\mathrm{AB}$ das equipes conforme localização rural e urbana, verificou-se maior percentual de padrão satisfatório $(45,3 \%)$ entre as equipes da zona urbana, em comparação com as equipes da zona rural (36,4\%). Na zona rural, nenhuma EqSF se classificou na categoria muito satisfatório, enquanto $6,3 \%$ da zona urbana obtiveram essa classificação, contudo, sem significância estatística $(\mathrm{p}=0,260)$. 
Tabela 2. Padrão de qualidade da Atenção Básica na dimensão Educação Permanente, Processo de Trabalho e Atenção Integral à Saúde, conforme localização urbana e rural. Equipes de Saúde da Família. Montes Claros, 2014

\begin{tabular}{lrrrc}
\hline \multirow{2}{*}{ Padrão de qualidade da Atenção Básica } & \multicolumn{2}{c}{ Urbana } & \multicolumn{2}{c}{ Rural } \\
\cline { 2 - 5 } & $\mathbf{n}$ & $\%$ & $\mathbf{n}$ & $\%$ \\
\hline Insatisfatório & 5 & 7,8 & 3 & 27,3 \\
Regular & 26 & 40,6 & 4 & 36,4 \\
Satisfatório & 29 & 45,3 & 4 & 36,4 \\
Muito satisfatório & 4 & 6,3 & 0 & 0,0 \\
\hline Total de Equipes & 64 & 100,0 & 11 & 100,0 \\
\hline
\end{tabular}

Likelihood Ratio: $p=0,260$.

A média de profissionais em cada EqSF foi $11,2( \pm 1,34)$, apresentando um crescimento linear em direção aos melhores padrões de qualidade da $\mathrm{AB}$, de 10,4 para $11,5, p=0,126$. A média de enfermeiros foi igual nas equipes, equivalente a um profissional e independentemente da classificação das equipes quanto ao padrão de qualidade. Com relação ao número médio de técnicos em enfermagem, a média geral foi menor que um profissional $(0,9)$ para cada equipe. Do mesmo modo, nem todas as equipes possuíam, pelo menos, um médico, um cirurgião-dentista e um auxiliar de odontologia, no ano da avaliação. Contudo, as equipes classificadas como 'muito satisfatório' apresentaram, pelo menos, um desses profissionais. Os agentes comunitários estiveram presentes em todas as equipes, independentemente do padrão de qualidade da $\mathrm{AB}$, com média de participação em cada equipe de 6,4 profissionais.

Tabela 3. Médias e desvio padrão de profissionais na Equipe de Saúde da Família conforme padrão de qualidade da Atenção Básica. Montes Claros, 2014

\begin{tabular}{|c|c|c|c|c|c|}
\hline \multirow{2}{*}{$\begin{array}{c}\text { Equipe de Saúde da Família } \\
\text { Composição profissional (Média } \pm \text { desvio padrão) }\end{array}$} & \multicolumn{5}{|c|}{$\begin{array}{l}\text { Padrão de qualidade da Atenção Básica na dimensão Educação Permanente, Processo de } \\
\text { Trabalho e Atenção Integral à Saúde }\end{array}$} \\
\hline & Insatisfatório & Regular & Satisfatório & Muito Satisfatório & Teste \\
\hline & \multicolumn{4}{|c|}{ Média (desvio padrão) } & $\mathrm{p}$ \\
\hline Profissionais em geral $(11,2 \pm 1,34)$ & $10,4( \pm 1,06)$ & $11,0( \pm 1,20)$ & $11,5( \pm 1,50)$ & $11,5( \pm 0,58)$ & 0,126 \\
\hline Enfermeiros $(1,0 \pm 0,16)$ & $1,0( \pm 0,00)$ & $1,0( \pm 0,00)$ & $1,0( \pm 0,00)$ & $1,0( \pm 0,00)$ & - \\
\hline Técnicos em enfermagem $(0,9 \pm 0,16)$ & $0,9( \pm 0,35)$ & $1,0( \pm 0,19)$ & $1,0( \pm 0,00)$ & $1,0( \pm 0,00)$ & 0,269 \\
\hline Médicos $(1,0 \pm 0,12)$ & $0,9( \pm 0,35)$ & $1,0( \pm 0,00)$ & $1,0( \pm 0,00)$ & $1,0( \pm 0,00)$ & 0,035 \\
\hline Cirurgiões-dentistas $(0,8 \pm 0,39)$ & $0,4( \pm 0,52)$ & $0,8( \pm 0,41)$ & $0,9( \pm 0,29)$ & $1,0( \pm 0,00)$ & 0,003 \\
\hline Auxiliares e técnicos da odontologia $(1,0 \pm 0,62)$ & $0,5( \pm 0,76)$ & $1,0( \pm 0,62)$ & $1,1( \pm 0,55)$ & $1,3( \pm 0,50)$ & 0,059 \\
\hline Agentes comunitários $(6,4 \pm 1,10)$ & $6,8( \pm 1,49)$ & $6,3( \pm 0,98)$ & $6,5( \pm 1,18)$ & $6,3( \pm 0,50)$ & 0,691 \\
\hline
\end{tabular}

Nas múltiplas comparações das médias, pelo teste Bonferroni, observou-se que a média de profissionais médicos foi diferente, de forma significativa, entre as equipes classificadas nos padrões de qualidade 'insatisfatório e regular' $(\mathrm{p}=0,036)$ e 'insatisfatório e satisfatório' $(\mathrm{p}=0,034)$. Para os cirurgiões-dentistas, a média de profissionais foi diferente entre as equipes classificadas como 'insatisfatório e regular' $(p=0,027)$, 
'insatisfatório e satisfatório' $(\mathrm{p}=0,002)$ e entre as equipes com padrão de qualidade 'insatisfatório e muito satisfatório' ( $\mathrm{p}=0,039)$.

Verificou-se, no teste de correlação, que o maior número de profissionais nas equipes, assim como mais médicos, mais cirurgiões-dentistas, mais auxiliares de odontologia, apresentou uma correlação linear positiva e significativa com a maior pontuação das equipes na dimensão, ou seja, melhor qualidade da $\mathrm{AB}$ na autoavaliação da equipe. Nesse sentido, $8,5 \%$ da variação na pontuação da dimensão podem ser explicados pelo número de profissionais em geral, sendo mais bem explicados pela variação do número de cirurgiões-dentistas (20,5\%), conforme tabela 4.

Tabela 4. Correlação entre pontuação das Equipes de Saúde da Família quanto ao padrão de qualidade da Atenção Básica e número de profissionais nas equipes. Montes Claros, 2014

\begin{tabular}{lccr}
\hline \multicolumn{1}{c}{ Equipe de Saúde da Família } & \multicolumn{2}{c}{$\begin{array}{r}\text { Pontuação da equipe na dimensão Educação Permanente, } \\
\text { Processo de Trabalho e Atenção Integral à Saúde }\end{array}$} \\
\hline \multicolumn{1}{c}{ Profissionais } & R & r2 & p \\
\hline Número de profissionais & 0,292 & 0,085 & 0,011 \\
Agentes Comunitários de Saúde & $-0,042$ & 0,001 & 0,721 \\
Médicos & 0,244 & 0,060 & 0,035 \\
Cirurgiões-dentistas & 0,453 & 0,205 & $<0,001$ \\
Técnicos/Auxiliares de odontologia & 0,333 & 0,110 & 0,003 \\
\hline
\end{tabular}

Com relação aos indicadores de saúde, verificou-se que as médias do número de pessoas com diabetes, hipertensão arterial sistêmica, doença de Chagas, hanseníase, tuberculose ou com alguma deficiência não apresentaram diferenças significativas conforme os diferentes padrões de qualidade entre as equipes. Contudo, observaram-se menores médias de pessoas com diabetes, hipertensão, hanseníase e com deficiências nas equipes classificadas no padrão 'insatisfatório’ para a qualidade da Atenção Básica.

Tabela 5. Médias e desvio padrão dos cadastros de agravos/doenças por Equipe de Saúde da Família conforme padrão de qualidade da Atenção Básica entre as equipes. Montes Claros, 2014

Cadastros por Equipe de Saúde da Família

\begin{tabular}{l} 
Agravos/doenças (Média \pm desvio padrão) \\
\hline Diabetes $(68,4 \pm 24,23)$ \\
Hipertensão Arterial Sistêmica $(338,4 \pm 98,03)$ \\
Doença de Chagas $(23,5 \pm 10,47)$ \\
Hanseníase $(0,8 \pm 0,97)$ \\
Pessoas com deficiência $(30,9 \pm 14,99)$ \\
Tuberculose $(0,8 \pm 3,00)$
\end{tabular}

Padrão de qualidade da Atenção Básica na dimensão Educação Permanente, Processo de Trabalho e Atenção Integral à Saúde

\begin{tabular}{rrrrr} 
Insatisfatório & Regular & Satisfatório & Muito Satisfatório & Teste \\
\hline \multicolumn{2}{c}{ Média (desvio padrão) } & & & $\mathrm{p}$ \\
\hline $51,5( \pm 20,13)$ & $70,9( \pm 24,05)$ & $69,7( \pm 24,32)$ & $73,3( \pm 27,71)$ & 0,218 \\
$285,4( \pm 96,43)$ & $339,1( \pm 98,60)$ & $352,3( \pm 102,17)$ & $324,0( \pm 11,05)$ & 0,383 \\
$24,1( \pm 12,43)$ & $23,0( \pm 11,84)$ & $23,7( \pm 9,56)$ & $24,5( \pm 3,79)$ & 0,986 \\
$0,5( \pm 1,07)$ & $0,7( \pm 0,79)$ & $0,8( \pm 0,96)$ & $1,5( \pm 1,92)$ & 0,391 \\
$20,0( \pm 9,06)$ & $29,5( \pm 14,01)$ & $33,9( \pm 15,90)$ & $38,3( \pm 15,69)$ & 0,077 \\
$0,0( \pm 0,00)$ & $0,6( \pm 0,73)$ & $1,4( \pm 4,41)$ & $0,0( \pm 0,00)$ & 0,512 \\
\hline
\end{tabular}




\section{Discussão}

O estudo contou com a participação de todas as EqSFs cadastradas no município que, em sua totalidade, aderiram ao PMAQ-AB e realizaram a autoavaliação. Os padrões de qualidade da $\mathrm{AB}$ entre as equipes na dimensão Educação Permanente, Processo de Trabalho e Atenção Integral à Saúde foram 'satisfatório' e 'muito satisfatório' para quase metade das equipes, e nenhuma se classificou no padrão 'muito insatisfatório'. Contudo, deve-se refletir acerca das equipes que foram classificadas como 'insatisfatório e regular', pois representam a grande parte das EqSFs do município. Esse resultado retrata presença de fragilidades e necessidade de adequações no modelo de assistência ofertado, para não comprometer a qualidade da $\mathrm{AB}$ e dar resolubilidade às demandas populacionais (SAVASSI, 2012).

Cabe destacar que, além dos processos de autoavaliação pelos sistemas municipais, há, também, a avaliação externa do Ministério da Saúde, prevista pelo PMAQ-AB. Os resultados das avaliações podem se desdobrar em incentivos financeiros para a gestão da saúde municipal, conforme o desempenho demonstrado (MOTA; DAVID, 2015), tornando-se necessário investir nas equipes que não alcançaram avaliações positivas, para que elas possam prestar uma assistência mais qualificada aos usuários.

A dimensão avaliada envolve, também, a autoanálise sobre educação permanente dos profissionais que compõem a EqSF. A educação permanente, baseada no aprendizado contínuo, é condição necessária para o desenvolvimento do sujeito, no que tange ao seu autoaprimoramento, direcionando-o à busca das competências pessoal, profissional e social, como uma meta a ser seguida por toda sua vida. É um compromisso pessoal a ser aprendido, conquistado com as mudanças de atitudes decorrentes das experiências vividas, por meio da relação com os outros, com o meio e com o trabalho (PASCHOAL; MANTOVANI; MÉIER, 2007).
Para que as ações e os serviços de saúde sejam mais efetivos com relação às necessidades de saúde da população, é preciso desenvolver, efetivamente, a educação permanente (LOPES ET AL., 2007). Nela, o trabalho torna-se o eixo fundamental da aprendizagem profissional. Os problemas comuns do processo de trabalho são essenciais para o desenvolvimento de experiências de aprendizagem coletiva (MERHY, 2015).

Ofertar cursos tradicionais, que negligenciam a aprendizagem no trabalho e o contexto local, não reflete em benefícios para o cotidiano dos serviços de saúde (COSTA-E-SILVA; RIVERA; HORTALE, 2007; NICOLETTO ET AL., 2009; SOUSA; MERCHÁN-HAMANN, 2009). Considera-se importante criar e manter espaços para a educação permanente em saúde (MATUMOTO ET AL., 2011) pelo planejamento coletivo de capacitações, a partir das discussões problematizadoras no contexto de trabalho (MARQUES ET AL., 2009; MEDEIROS ET AL., 2010; CAVALCANTI; PADILHA, 2014). A educação permanente trata-se de política de gestão de serviços, com objetivo de alcançar tanto resolutividade como, também, integralidade na assistência humanizada à saúde (CAVALCANTI; PADILHA, 2014). Assim, os resultados da autoavaliação das EqSFs poderão nortear o processo de educação permanente, com maior atenção para as equipes que apresentaram padrões inadequados de qualidade para a dimensão avaliada.

Implementar processos de ensino e aprendizagem respaldados por ações crítico-reflexivas e participativas é um desafio, contudo, são importantes para promover mudanças na realidade local de cada serviço. As composições saúde e educação e trabalho e educação apresentam dificuldades diversas relacionadas com infraestrutura material, mas que perpassam, também, as fragilidades da gestão e de recursos humanos necessários para desenvolver e aplicar as ações de educação permanente (MICCAS; BATISTA, 2014).

Neste estudo, a maioria das equipes encontrava-se localizada na zona urbana de Montes Claros. Entre as equipes situadas na 
zona rural, mais da metade foi classificada nos padrões 'insatisfatório' ou 'regular', e nenhuma equipe foi classificada como 'muito satisfatório’ na dimensão avaliada. Apesar da não associação estatística entre localização e qualidade $\mathrm{da} A B$ entre as equipes, cabe ressaltar a maior frequência de autoavaliações negativas para as equipes da zona rural, que apresentaram piores níveis de qualidade da $\mathrm{AB}$.

Na zona rural, uma pior avaliação também pode ser explicada pelo quesito Assistência Integral à Saúde, que compõe o processo avaliativo da dimensão analisada neste estudo. Conforme a Lei $\mathrm{n}^{0} 8.080 / 90$ - Lei Orgânica da Saúde, em seu Art. $7^{\circ}$, inciso II -, a integralidade da assistência é entendida como um conjunto articulado e contínuo das ações e dos serviços preventivos e curativos, individuais e coletivos, exigidos para cada caso em todos os níveis de complexidade do sistema (BRASIL, 1990). A integralidade da atenção envolve relação de cuidado e comprometimento com o processo e as pessoas (LOPES ET AL., 2007).

Nessa perspectiva, é possível supor que a distância geográfica entre a residência do usuário e a unidade de saúde da ESF é fator que interfere no acesso aos serviços de saúde e na integralidade da atenção. Ademais, a dificuldade de o usuário obter assistência em outros níveis de atenção, secundário e terciário, localizados na zona urbana do município, é outro fator que merece ser discutido no âmbito da ESF. Assim, é possível sugerir que as barreiras geográficas são fatores que interferem no acesso aos serviços de saúde e fragilizam a assistência integral.

Aponta-se o termo acessibilidade como o elemento estrutural necessário para a primeira atenção e dependente de o local de atendimento ser acessível do ponto de vista da facilidade e da disponibilidade. Caso isso não seja factível, haverá postergação na atenção, podendo, inclusive, comprometer o diagnóstico e o manejo do problema (STARFIELD, 2002).

Existem diferentes tipos de acessibilidade, e pode-se distinguir acesso sócio-organizacional de acesso geográfico. O primeiro inclui recursos que facilitam ou dificultam os esforços das pessoas para chegarem ao atendimento, como idade, raça, classe social ou alguma forma de pagamento. $\mathrm{O}$ acesso geográfico, por outro lado, envolve as características relacionadas à distância e ao tempo para alcançar e obter os serviços (SCHWARTZ, 2010). No atual estudo, sugere-se que a limitação do acesso geográfico é que pode ter contribuído para uma classificação negativa de qualidade da $\mathrm{AB}$ entre as equipes localizadas na zona rural, na dimensão avaliada da AMAQ.

Para além da questão do acesso geográfico, as dificuldades de acesso à saúde, nas unidades básicas, podem ser atribuídas a fatores como desorganização do sistema de referência-contrarreferência (OLIVEIRA; MATTOS; SOUZA, 2009). Nesse sentido, pode-se inferir que os moradores da zona rural, assistidos por uma EqSF, apresentarão uma maior dificuldade no uso dos serviços referenciados, pois, além da falta de organização da rede de saúde pública, eles se encontram mais distantes dos serviços especializados, como discutido anteriormente.

Uma pesquisa conduzida no âmbito da saúde da família, do mesmo município do atual estudo, constatou que os profissionais de saúde percebem fragilidades na comunicação entre os diferentes níveis da rede do Sistema Único de Saúde (SUS), fato que sugere fragmentação no modelo de saúde, no acesso e na atenção integral (COSTA ET AL., 2013).

Entretanto, destaca-se o princípio da igualdade exposto na Lei Orgânica da Saúde - todo cidadão brasileiro tem direito à assistência em saúde (BRASIL, 1990). Assim, o sistema público deve garantir ações e serviços de saúde para os brasileiros, de acordo com as necessidades de cada um, reduzindo as disparidades sociais e regionais; no caso, fornecer estrutura adequada para a locomoção de pessoas, da zona rural, referenciadas aos outros níveis da rede de saúde.

O número médio de profissionais em cada 
EqSF foi correspondente ao previsto na legislação brasileira. Contudo, as melhores classificações e pontuações foram as das equipes com mais profissionais. Observou-se que nem toda equipe era composta por profissional médico, cirurgião-dentista e técnico/auxiliar de odontologia. Aquelas equipes com mais profissionais nessas categorias apresentaram melhores pontuações, o que sugere que a equipe multiprofissional completa obtém melhores resultados no processo de trabalho e na atenção integral à saúde.

De acordo com a nova Política de Atenção Básica, proposta pela Portaria no ${ }^{0} .488 / 2011$, além da composição básica: um médico, um enfermeiro, um auxiliar de enfermagem e seis agentes comunitários de saúde; inclui-se a equipe de saúde bucal nas EqSFs, acrescentando, assim, um cirurgião-dentista, um técnico em saúde bucal e um auxiliar em saúde bucal (BRASIL, 2011). Criar uma equipe de profissionais de saúde é um processo desafiador, que se relaciona com as definições políticas e com as práticas das instituições no atendimento às expectativas sobre a qualidade da atenção prestada à comunidade (RODRIGUEZ; POZZEBON, 2010). Condições favoráveis melhoram a possibilidade de uso dos serviços de saúde com resolubilidade na $\mathrm{AB}$ (ALVES ET AL., 2014). Os resultados deste estudo demonstraram que as equipes completas, em número e em categorias profissionais, obtiveram melhores classificações na autoavaliação de qualidade da $\mathrm{AB}$.

Sobre a relação entre integralidade da atenção à saúde e educação permanente, pode-se afirmar que a integralidade é eixo norteador da proposta de educação permanente em saúde, porque direciona o trabalho em saúde para um trabalho multidisciplinar e multiprofissional. No trabalho em equipe multidisciplinar, estabelece-se o diálogo entre os profissionais de diversas áreas para solucionar um problema. Dessa forma, a integralidade envolve a compreensão dos problemas de saúde em suas diferentes dimensões. A atenção integral traz a possibilidade de formulação de políticas de formação e desenvolvimento dos trabalhadores do SUS, coerentes com a acolhida e a responsabilidade do conjunto integrado do sistema. Envolve relação de cuidado e comprometimento com o processo de trabalho e as pessoas (LOPES ET AL., 2007).

Quanto aos cadastros de agravos/doenças pelas equipes, as médias de pessoas com diabetes, hipertensão arterial, doença de Chagas, hanseníase, tuberculose e com alguma deficiência não se diferenciaram de forma estatisticamente significativa, conforme os diferentes padrões de qualidade da $\mathrm{AB}$ entre as equipes. Fato interessante, uma vez que a pior qualidade da $A B$ poderia representar uma área territorial de assistência composta por população menos saudável. Isso porque a dimensão avaliada da AMAQ envolve autoanálise da equipe quanto ao processo de trabalho e à atenção integral à saúde. Mesmo que os resultados do atual estudo não tenham demonstrado relação entre melhores padrões de qualidade da $\mathrm{AB}$ com menor número de cadastros de agravos/ doenças por equipe, cabe salientar a importância da vigilância epidemiológica nos territórios de abrangência da saúde da família.

A vigilância é uma importante ferramenta para planejar ações de prevenção e controlar doenças em saúde pública, e que poderia impactar em melhor qualidade da AB. Na ESF, adentrar o cotidiano da população requer dos profissionais de saúde um olhar qualificado para a identificação dos problemas de saúde. Os cuidados dirigidos a uma população específica incluem, além da atenção ao indivíduo, a vigilância dos problemas mais importantes e seus determinantes; o planejamento das intervenções preventivas e terapêuticas mais efetivas para a população; e um movimento para a melhoria dos níveis de saúde e das condições de vida das pessoas (SARTI ET AL., 2012).

Quanto às limitações deste estudo, deve-se destacar a possibilidade de vieses de informação, uma vez que se utilizaram dados 
do Siab, passíveis de erros, como a subnotificação dos cadastros de agravos/doenças pelas EqSFs.

\section{Conclusões}

Ao avaliar a qualidade da $\mathrm{AB}$ pela dimensão Educação Permanente, Processo de Trabalho e Atenção Integral à Saúde, constatou-se que as melhores autoavaliações se referiram às equipes localizadas na zona urbana, apesar da não diferença estatística, quando comparadas às rurais. Incorporação de mais trabalhadores e ampliação das categorias profissionais, pela inclusão da área médica e odontológica, melhoram a autoavaliação das EqSFs. Dados das equipes relacionados aos cadastros de agravos/doenças não se mostraram diferentes nos distintos padrões de qualidade da $\mathrm{AB}$ entre as equipes.

Considera-se que a autoavaliação das
EqSFs seja o ponto de partida no processo de reorganização do processo de trabalho e da gestão em saúde. Pela problematização e pelo reconhecimento de fatores, como localização geográfica, composição da equipe e indicadores de saúde, pode-se planejar intervenções e construir estratégias concretas para superar os problemas identificados, bem como garantir melhores avaliações da $A B$ na visão dos profissionais de saúde.

\section{Colaboradores}

Moreira, K. S.; Vieira, M. A.; Costa, S. M. contribuíram substancialmente para a concepção, o planejamento e para a análise e a interpretação dos dados; contribuíram significativamente na elaboração do rascunho e na revisão crítica do conteúdo; e participaram da aprovação da versão final do manuscrito.

\section{Referências}

ALVES, M. G. M. et al. Fatores condicionantes para o acesso às equipes da Estratégia Saúde da Família no Brasil. Saúde debate, Rio de Janeiro, v. 38 n. esp., p. 3451, out. 2014.

BRASIL. Ministério da Saúde. Lei nº 8.080, de 19 de setembro de 1990. Dispõe sobre as condições para a promoção, proteção e recuperação da saúde, a organização e o funcionamento dos serviços correspondentes e dá outras providências. Diário Oficial [da] União. Brasília, DF, 19 set. 1990. Disponível em: <http://conselho.saude.gov.br/legislacao/ lei8080_190990.htm>. Acesso em: 21 nov. 2016.

\section{Ministério da Saúde. Programa Nacional de}

Melhoria do Acesso e da Qualidade da Atenção Básica

(PMAQ). Brasília, DF: Ministério da Saúde: 2011.

Disponível em: $<$ http://dab.saude.gov.br/portaldab/

ape_pmaq.php>. Acesso em: 21 nov. 2016.

CAVALCANTI, Y. W.; PADILHA, W. W. N. Qualificação

de processos de gestão e atenção no município

de Caaporã, PB: relatos de tutoria de educação

permanente em saúde. Saúde debate, Rio de Janeiro, v. 
38, n. 100, p. 170-180, mar. 2014.

COSTA, S. M. et al. Referência e contrarreferência na saúde da família: percepção dos profissionais de saúde. Rev APS, Juiz de Fora, v. 16, n. 3, p. 287-293, set. 2013.

COSTA-E-SILVA, V.; RIVERA, F. J. U.; HORTALE, V. A Projeto Integrar: avaliação da implantação de serviços integrados de saúde no Município de Vitória, Espírito Santo, Brasil. Cad. Saúde Pública, Rio de Janeiro, v. 23, n. 6, p. 1405-1414, jun. 2007.

\section{INSTITUTO BRASILEIRO DE GEOGRAFIA E} ESTATÍSTICA (IBGE). Censo demográfico: resultados gerais da amostra. Rio de Janeiro: IBGE: 2015. Disponível em: <ftp://ftp.ibge.gov.br/Estimativas_ de_Populacao/Estimativas_2015/estimativa_2015_ TCU_20160712.pdf>. Acesso em: 21 nov. 2016.

KAWATA, L. S. et al. O trabalho cotidiano da enfermeira na saúde da família: utilização de ferramentas da gestão. Texto contexto - enferm., Santa Catarina, v. 18, n. 2, p. 313-320, abr./jun. 2009.

LOPES, S. R. S. et al. Potencialidades da educação permanente para a transformação das práticas em saúde. Com. Ciências Saúde, Brasília, DF, v. 18, n. 2, p. 147-155, jun. 2007.

MARQUES, E. S. et al. Práticas e percepções acerca do aleitamento materno: consensos e dissensos no cotidiano de cuidado numa Unidade de Saúde da Família. Physis, Rio de Janeiro, v. 19, n. 2, p. 439-455, 2009.

MATUMOTO, S. et al. A prática clínica do enfermeiro na atenção básica: um processo em construção. Rev Latino-Am Enfermagem, Ribeirão Preto, v. 19, n. 1, p. 123-130, jan./fev. 2011.

MEDEIROS, A. C. et al. Gestão participativa na educação permanente em saúde: olhar das enfermeiras. Rev Bras Enferm., Brasília, DF, v. 63, n. 1, p. 38-42, jan./ fev. 2010

MERHY, E. E. Educação permanente em movimento: uma política de reconhecimento e cooperação, ativando os encontros do cotidiano no mundo do trabalho em saúde, questões para os gestores, trabalhadores e quem mais quiser se ver nisso. Saúde em Redes, Porto Alegre, v. 1, n. 1, p. 7-14, 2015 .

MICCAS, F. L.; BATISTA, S. H. S. S. Educação permanente em saúde: metassíntese. Rev. Saúde Pública, São Paulo, v. 48, n. 1, p. 170-185, fev. 2014.

MOTA, R. R. A.; DAVID, H. M. S. L. Programa Nacional de Melhoria do Acesso e da Qualidade da Atenção Básica: questões a problematizar. Rev. enferm. UERJ, Rio de Janeiro, v. 23, n. 1, p. 122-127, jan./fev. 2015.

NICOLETTO, S. C. S. et al. Polos de educação permanente em saúde: uma análise da vivência dos atores sociais no norte do Paraná. Interface (Botucatu), Botucatu, v. 13, n. 30, p. 209-219, jul./set. 2009.

OLIVEIRA, L. H.; MATTOS, R. A.; SOUZA, A. I. S. Cidadãos peregrinos: os "usuários" do SUS e os significados de sua demanda a prontos-socorros e hospitais no contexto de um processo de reorientação do modelo assistencial. Ciênc. saúde coletiva, Rio de Janeiro, v. 14, n. 5, p. 1929-1938, nov./dez. 2009.

PASCHOAL, A. S.; MANTOVANI, M. F.; MÉIER, M. J. Percepção da educação permanente, continuada e em serviço para enfermeiros de um hospital de ensino. Esc Enferm USP., São Paulo, v. 41, n. 3, p. 478-484, set. 2007.

RODRIGUEZ, C.; POZZEBON, M. The implementation evaluation of primary care groups of practice: a focus and organization identity. BMC Fam Pract. London, v. 11, n. 15 , fev. 2010

SARTI, T. D. et al. Avaliação das ações de planejamento em saúde empreendidas por equipes de saúde da família. Cad Saúde Pública, Rio de Janeiro, v. 28, n. 3, p. 537-548, mar. 2012.

SAVASSI, L. C. M. Qualidade em serviços públicos: os desafios da atenção primária. Rev bras med fam comunidade, Ouro Preto, v. 7, n. 23, p. 69-74, abr./jun. 2012. 
SCHRAIBER, L. B. et al. Planejamento, gestão e avaliação em saúde: identificando problemas. Ciênc. saúde coletiva, Rio de Janeiro, v. 4, n. 2, p. 221-242, 1999.

SCHWARTZ, T. D. Estratégia Saúde da Família: avaliando o acesso ao SUS a partir da percepção dos usuários da Unidade de Saúde de Resistência, na região de São Pedro, no município de Vitória (ES). Ciênc. saúde coletiva, Rio de Janeiro, v. 15, n. 4, p. 2145-2154, jul. 2010.

SOUSA, M. F.; MERCHÁN-HAMANN, E. Saúde da Família no Brasil: estratégia de superação da desigualdade na saúde? Physis, Rio de Janeiro, v. 19, n. 3, p. 711-729, 2009.

\section{STARFIELD, B. Atenção primária: equilíbrio entre} necessidades de saúde, serviços e tecnologia. Brasília, DF: Unesco; Brasília, DF: Ministério da Saúde; 2002.

Recebido para publicação em agosto de 2016

Versão final em novembro de 2016

Conflito de interesses: inexistente

Suporte financeiro: não houve 